\title{
Spectroscopic modelling of four neutron star low-mass X-ray binaries using CLOUDY
}

\author{
Gargi Shaw $^{1}{ }^{\star}$ Sudip Bhattacharyya ${ }^{1}$ \\ ${ }^{1}$ Department of Astronomy and Astrophysics, Tata Institute of Fundamental Research, \\ Homi Bhabha Road, Navy Nagar, Colaba, Mumbai 400005, India
}

Accepted for publication in MNRAS

\begin{abstract}
Low-mass X-ray binaries (LMXBs) have a wide range of X-ray properties which can be utilised to reveal many physical conditions of the associated accretion discs. We use the spectral synthesis code CLOUDY to perform a detailed modelling of neutron star LMXBs GX 13+1, MXB 1659-298, 4U 1323-62 and XB 1916-053; and characterise the underlying physical conditions, such as density, radiation field, metallicity, wind velocity, etc. For this purpose we model highly ionised spectra of $\mathrm{Fe}, \mathrm{Ca}, \mathrm{S}, \mathrm{Si}, \mathrm{Mg}, \mathrm{Al}$ in the soft X-ray band, and compare the predicted line flux ratios with the observed values. We also find that the strength and profile of these spectral lines get modified in the presence of magnetic field in the accretion disc. Using this, we estimate an upper limit of the existing magnetic field to be about a few hundred to a few thousand $\mathrm{G}$ in the accretion discs of these four LMXBs.
\end{abstract}

Key words: accretion, accretion disc - magnetic fields - stars: neutron - techniques: spectroscopic - X-rays: binaries: individual (GX 13+1, MXB 1659-298, 4U 1323-62, XB 1916-053)

\section{INTRODUCTION}

A low-mass X-ray binary (LMXB) system consists of a neutron star or a black hole, and a companion star with a mass similar to or less than the Sun. The companion star transfers its mass by Roche-lobe overflow, in which the material initially gets pulled into an accretion disc around the neutron star or the black hole and then slowly spirals into the enormous gravitational well of the compact object. The accreted material is heated up to a very high temperature causing the system to shine brightly in X-rays (Shapiro \& Teukolski 1984; Tauris \& Heuvel 2006).

High-inclination LMXBs usually show absorption lines in the X-ray spectrum, as the line-of-sight passes through the structures above the accretion disc in such cases. In fact iron $\mathrm{K} \alpha$ fluorescence lines, Fe XXV (6.700 keV) and Fe XXVI (6.966 keV), have been observed from many highinclination LMXBs, such as X 1254-690, MXB 1658-298, X 1624-490 (Díaz Trigo et al. 2006), XTE J1710-281 (Younes et al. 2009) and 4U 1916-053 (Díaz Trigo et al. 2006). In addition to these Iron $\mathrm{K} \alpha$ lines, many other highly ionised lines for Mg XII (1.472 keV), Al XIII (1.728 keV), Si XIV (2.005 keV), S VI (2.621 keV), Ca XX (4.105keV), Ne X $(1.0218 \mathrm{keV})$, O VIII $(0.6536 \mathrm{keV})$ have been observed from

* E-mail: gargishaw@gmail.com (GS) some LMXBs, such as GX 13+1 (D'Aì et al. 2014) and MXB 1659-298 (Iaria et al. 2018b). These lines contain a wealth of information, and hence can be used to decipher the underlying physical conditions which are responsible for their origin. In this paper we aim to study four neutron star LMXBs, GX 13+1, MXB 1659-298, 4U 1323-62 and XB 1916-053, by detailed spectroscopic modelling.

The LMXB 4U 1323-62 was first observed with (van der Klis et al. 1985) the UHURU satellite. It has the orbital period of 2.94 hour (Boirin et al. 2005) (hereafter B05) and shows $1 \mathrm{~Hz}$ quasi-periodic oscillation (QPO) and frequent thermonuclear bursts. Simultaneous intensity dips and bursts in X-rays were also observed from this source (van der Klis et al. 1985). Later, B05 observed highly ionised Fe XXV and Fe XXVI lines at $6.68 \pm 0.04 \mathrm{keV}$ and $6.97 \pm 0.05$ $\mathrm{keV}$ respectively, and also the change in intensity for the same lines, with XMM-Newton. They estimated the angle between the line-of-sight and the rotational axis of the accretion disc to be approximately 60 degrees. On the other hand, XB 1916-053 was first observed by White \& Swank (1982) with a orbital period of $3000.6 \pm 0.2 \mathrm{sec}$ (Homer et al. 2001). It is $9.3 \mathrm{kpc}$ away (Foulkes et al. 2010) and the angle between the line-of-sight and the rotational axis of the accretion disc is approximately 77 degrees. MXB 1659-298 was first observed by Lewin et al. (1976). Recently, Iaria et al. (2018b) have observed several highly ionised lines, such 
as Ne X, O VIII, Fe XXV and Fe XXVI, and estimated an inclination angle of $72 \pm 3$ degrees (Iaria et al. 2018a). GX $13+1$ is another important LMXB which shows many highly ionised absorption lines. It is one of the brightest Galactic LMXBs, which is at a distance of $7 \pm 1 \mathrm{kpc}$. It has the longest known orbital period for a Galactic neutron star LMXB, and it has a K5III spectral type donor star (Bandyopadhyay et al. 1999). GX 13+1 was first observed by Fleischman (1985) using HEAO 1 satellite. Later a periodicity of 24.27 days was observed in its power spectrum density obtained from data collected over 14 years with the All Sky Monitor (ASM) on board the Rossi X-ray Timing Explorer (RXTE) (Iaria et al. 2013). Though it has been classified as an atoll source, it shows properties that are close to the $\mathrm{Z}$ sources and several properties still remain unexplained. Moreover, GX 13+1 shows thermonuclear bursts and super bursts in X-ray, and it also shows a QPO at $61 \pm 1 \mathrm{~Hz}$ (D'Aì et al. 2014). D'Aì et al. (2014) have also observed dips for a duration of 450 secs. The inclination angle of the source is $>65^{\circ}$. A wealth of $\mathrm{X}$-ray spectroscopic observations is available for GX 13+1, which includes Fe XXV, Fe XXVI, Mg XII, Al XIII, Si XIV, $\mathrm{S}$ VI, Ca XX absorption lines in the persistent phase (D'Aì et al. 2014).

These absorption lines from the above mentioned sources are useful to decipher the chemical, physical as well as kinematic properties of the accretion structure. We use the spectral simulation code, CLOUDY, in this work to model iron $\mathrm{K} \alpha$ fluorescence lines, Fe XXV and Fe XXVI, from two LMXBs, 4U 1323-62 and XB 1916-053 and also other highly ionised lines like Mg XII, Al XIII, Si XIV, S XVI , Ca XX, Ne X, O VIII from GX 13 +1 (D'Aì et al. 2014) and MXB 1659-298 (Iaria et al. 2018b). We mainly focus on spectroscopic modelling of these highly ionised lines in the soft X-ray band and determine the underlying physical conditions which give rise to these lines.

Moreover, such a large number of absorption lines could also be analysed in a way such that they can help to probe the magnetic field in the accretion disc. So far, to our best knowledge, no one has estimated the strength of magnetic field or its upper limit in the accretion discs of $4 \mathrm{U} 1323$ 62, XB 1916-053, MXB 1659-298 and GX 13+1. We take this opportunity to constrain the magnetic fields for these accretion discs.

Here, we mention some important roles of disk magnetic field. In accretion discs, magnetic field is an important source of viscosity. Weakly-magnetised differentiallyrotating accretion discs are generally unstable and shows magnetohydrodynamical instability and magnetohydrodynamical turbulence (Balbus \& Hawley 1994), which could give rise to outbursts. Many accreting systems show powerful jets and it is believed that the magnetic field couples discs and jets in such systems. Highly collimated jets in black hole binaries could be explained by strong magnetic field existing in the inner accretion disc (McKinney \& Narayan 2007). However, the origin of accretion disc magnetic field is not well understood yet. In case of an accreting neutron star with a magnetosphere, it is very natural to expect that the surrounding accretion disc will also harbour some amount of magnetic field (Naso et al. 2013). This may be due to threading of neutron star magnetosphere and its own magnetic field. Perfrey et al. (2016) numerically showed that the interaction of neutron star magnetic field with its dif- ferentially rotating accretion disc gives rise to opening of pulsar magnetic field lines. This could be tested if the disc magnetic field can somehow be measured. It is thus very important to estimate the magnetic field in accretion discs. Silantev et al. (2013) have estimated magnetic fields of active galactic nuclei (AGN) using polarised broad $\mathrm{H} \alpha$ lines. They used wavelength dependence of polarisation degree inside this line to measure magnetic field, and found $\mathrm{B}_{\|}=0$, $\mathrm{B}_{\phi}=14 \mathrm{G}$ for the AGN Akn 120 and $\mathrm{B}_{\|}=0.6 \mathrm{G}, \mathrm{B}_{\phi}=8.5 \mathrm{G}$ for Mrk 6, respectively. Linear polarimetric observations in near-infrared and optical band of LMXB jets were done by Russel \& Fender (2018), but they did not calculate magnetic field strength. In fact, so far, to the best of our knowledge, the accretion disc magnetic field for LMXBs has not been measured directly. The strength of this field is not expected to be high enough to produce a detectable Zeeman splitting. In future, X-ray polarimetry could be used to estimate such a field.

In this work, along with modelling the observed lines in the soft X-ray band to determine physical parameters of the accretion discs, we also show that it is possible to estimate an upper limit of the magnetic field in an accretion disc using the absorption lines. We apply this method for $4 \mathrm{U}$ 1323-62, XB 1916-053, MXB 1659-298 and GX 13+1.

This paper is organised as follows. In section 2, we describe our calculations. Results for the LMXBs 4U 1323-62, 4U 1916-053, MXB 1659-298, GX 13+1, and our conclusions are presented in sections 3 and 4 respectively.

\section{CALCULATIONS}

\subsection{Numerical Methods}

In this section, we present our calculations with the detailed method which are carried out using the spectral synthesis code CLOUDY (Ferland et al. 2013, 2017). It is a micro-physics code based on a self-consistent $a b$ initio calculation of thermal, ionisation, and chemical balance of nonequilibrium gas and dust exposed to a source of radiation. This software and its documentation is freely available at $h t t p: / / n u b l a d o . o r g$, and it is widely used as one of the astrophysical plasma codes. It can be utilised to model emission lines in various astrophysical environments, from gaseous nebulae to quasars covering a wide range of temperature and density. It predicts column densities of various species and resultant spectra ranging from gamma rays to radio and vice versa using a minimum number of input parameters like density, radiation field, chemical composition and geometry. There are various options to make a simulated model to the most realistic one. Currently CLOUDY includes 625 species including atoms, ions and molecules and uses five distinct databases: H-like and He-like iso-electronic sequences (Porter et al. 2012), the $\mathrm{H}_{2}$ molecule (Shaw et al. 2005), Stout, CHIANTI (Landi et al. 2012) and LAMBDA (Schoier et al. 2005) to model spectral lines. Atoms of the $\mathrm{H}$-like iso-electronic sequence have one bound electron and atoms of the He-like iso-electronic sequence have two bound electrons. CLOUDY uses an unified model for both the $\mathrm{H}-$ like and He-like iso-electronic sequences, that extends from $\mathrm{H}$ to $\mathrm{Zn}$, as described by Porter et al. (2012). The highly ionised lines modelled here belong to either $\mathrm{H}$-like or He-like 
iso-electronic sequences. O VIII, Ne X, Ca XX, Fe XXVI, $\mathrm{S}$ XVI, Si XIV, Mg XII, Al XIII have H-like iso-electronic sequences, whereas Fe XXV has He-like iso-electronic sequence. Any number of levels up to 400 can be computed and the ionisation and level populations are self-consistently determined by solving the full collisional-radiative problem. Increasing the number of levels allows a better representation of the collision physics that occurs within higher levels of the atom but it consumes more time and memory. In the default mode of CLOUDY, levels up to 50 are included. We have included default number of levels in our calculations. Earlier, various research groups, for example, B05 and Nielsen \& Gilfanov (2015), used CLOUDY to model LMXBs. Here we aim to use the version c-17 of CLOUDY which is more advanced than the earlier versions and our models incorporate more parameters than that were included previously which will be discussed in this section.

For all of our models, we assume a geometry of a constant pressure clumpy highly-ionised gaseous rotating disc around a 1.4 solar mass neutron star with high velocity wind. Earlier groups have neither included clumpiness nor the rotating disc around a 1.4 solar mass neutron star with high velocity wind. Here hydrogen density, incident radiation field, metallicity, clumpiness and wind are model parameters whose values can be varied. Below we discuss our input parameters briefly.

For hydrogen density $n_{H}\left(\mathrm{~cm}^{-3}\right)$, we use the total hydrogen density and is given by

$n_{H}=n_{H_{0}}+n_{H^{+}}+2 n_{H_{2}}+\sum_{\text {other }} n_{H_{\text {other }}}$,

where $n_{H_{0}}, n_{H^{+}}, 2 n_{H_{2}}$ and $n_{H_{\text {other }}}$ represent $\mathrm{H}$ in neutral, ionised, hydrogen molecule and all other hydrogen-bearing molecular states, respectively. One can vary the density spatially in different ways across the whole gaseous extent. We use following radius dependent power law density profile for all our models presented here,

$n_{H}(r)=n_{H}\left(r_{0}\right) \times\left(r / r_{0}\right)^{\alpha}$.

Here $n_{H}(r), n_{H}\left(r_{0}\right)$ are the density at a distance $r$ and at the illuminated face at $r_{0}$, respectively. The power law index $\alpha$ can be varied. Previous modellers (Boirin et al. 2005) used the simplest plane parallel geometry. In a plane parallel geometry, the ratio of thickness to the inner radius $\left(r-r_{0}\right) / r_{0}$ is $<0.1$. Hence, for a plane parallel model, the value of power law index does not affect the results. However, for a thick shell model, where $\left(r-r_{0}\right) / r_{0}$ is $\left.<3\right)$, the results do depend on the value of power law index. Here, we consider a more realistic thick shell geometry and we use a power law index of $-15 / 8$ (Frank et al. 2002).

Tanaka, Y (1997) showed that the X-ray spectrum of LMXBs can be of following 4 types : i) hard power law with a sharp cut-off above a few tens of keV, ii) soft thermal component accompanied by a hard tail, iii) thermal bremsstrahlung, and iv) a single power law. Here we assume that the gaseous disc is exposed to incident radiation coming from a thermal blackbody and a single power law due to Comptonisation, following Juett \& Chakrabarty (2005) and B05. We use ionisation parameter $\xi\left(\mathrm{erg} \mathrm{cm} \mathrm{s}^{-1}\right)$ to quantify this radiation, and it is given by,

$\xi=\frac{L_{i o n}}{n(H) r^{2}}=(4 \pi)^{2} \int_{1 R}^{1000 R} \frac{J_{\nu} d \nu}{n(H)}$.
Here $J_{\nu}$ is the mean intensity in erg s $\mathrm{s}^{-1} \mathrm{sr}^{-1} \mathrm{~Hz}^{-1}$. The integration limit ranges from $R$ to $1000 R$ in units of Ry, Ry being the ionization energy of hydrogen atom. Following Kallman \& Bautista (2001), we include all ionising radiation, integrating over all ionising photon energies from $1 R$ to $1000 R$. The source-gaseous-disc separation is represented by $r . L_{i o n}$ is the luminosity between 1 and $10^{3} \mathrm{Ry}$.

Boirin et al. (2005) and earlier modellers assumed plane parallel geometry without any wind. We find that a rotating disc model produces smaller $\chi^{2}$ than a plane parallel geometry, and hence, we assume a rotating disc with wind in all our calculations presented here. For a rotating disc geometry, the inward gravitational acceleration is calculated as

$g=\frac{G M}{r^{2}}\left(1-\frac{r_{0}}{r}\right)$,

where $r_{0}$ is the inner radius. In this given set up, CLOUDY evaluates the line widths and escape probabilities of photons in the Sobolev or Large Velocity Gradient approximation (Sobolev 1957). The effective line optical depth is given by

$\tau_{l, r}=\alpha_{l, u} \min (r, \Delta r)\left(n_{l}-n_{u} \frac{g_{l}}{g_{u}}\right)\left(\frac{u_{t h}}{\max \left(u_{t h}, u_{\exp }\right)}\right)$.

Here, $n_{l}, n_{u}, g_{l}, g_{u}$ and $\alpha_{l, u}$ are level populations and statistical weights in lower and upper levels and line absorption co-efficient, respectively. $u_{t h}$ and $u_{\text {exp }}$ are the thermal and expansion velocities respectively, and the radius used is the smaller of the depth $(\Delta r)$ or the radius $(r)$.

Other modellers, whom we mentioned earlier, ignored the magnetic field in their calculations. However, as we mentioned in the introduction section that the effect of magnetic fields can be important in accretion discs, we include the magnetic field in our models as one of the input parameters, and study its effect on highly ionised soft x-ray lines. Here, as mentioned below, we suggest a plausible way to estimate the upper limit of LMXB disc magnetic field. It is known that the magnetic field cools high-temperature ionised gas through cyclotron emission and takes dominant part in thermal cooling and contributes to gas pressure. Hence this field is capable to change the strength and profile of emission or absorption lines from a high-temperature ionised gas. Therefore, the strengths and profiles of lines originating in accretion discs can be utilised to estimate an upper limit of the local magnetic field. Such a field prevailing in the accretion discs can be either ordered or tangled. We do not include ordered magnetic field in our calculation as the angle between the radiation field of the central object and the magnetic field is unknown. Our used tangled magnetic field (in units of $\mathrm{G}$ ) follows the equation

$B=B_{0} \times\left(n_{H}(r) / n_{H}\left(r_{0}\right)\right)^{\gamma / 2}$.

Here, $B_{0}$ is a free parameter and the term in parenthesis is the ratio of the density at a distance $r$ to the density at the illuminated face of the gaseous disc at $r_{0} . \gamma$ depends on geometry and for the tangled field we use the default value provided by CLOUDY, $\gamma=4 / 3$.

All the models calculated here are constant pressure models. The total pressure consists of ram, magnetic, turbulent, particle, and radiation pressures. The magnetic pressure and the enthalpy terms are also considered in these models.

CLOUDY requires at least one stopping condition to 
complete its calculation. Here, we use ionised column density as one of the stopping conditions for our calculations. No observed ionised column density is reported yet for the above mentioned systems, so we vary the ionised column density in our calculations. Our other stopping condition is the thickness of the accretion disc. CLOUDY stops calculation as soon as it satisfies any one of these two conditions mentioned. Best model is calculated by using a built-in optimisation program based on phymir algorithm (van Hoof 1997) which calculates a non-standard goodness-of-fit estimator $\chi^{2}$ and minimises it by varying input parameters. This optimisation program is user friendly and preferred by many astronomers (Ferland et al. 2013; Srianand \& Petitjean 2000; van Hoof et al. 2013; Rawlins et al. 2018). We try to get best model parameters by varying total hydrogen density, ionisation parameters, metallicity, clumpiness, magnetic field and ionised column density. CLOUDY executes detailed radiative transfer computations and produces line fluxes, column densities of various species, heating and cooling, self-consistently. In the following, we show the effect of important parameters on the Fe XXV and Fe XXVI lines, since these lines are present in all the four LMXBs modelled here. Other highly ionised lines mentioned in this work also show similar effects with different extents.

\subsection{Effect of density}

First, we consider a sample model for LMXBs where we assume a geometry of a constant pressure clumpy highlyionised gaseous rotating disc around a 1.4 solar mass neutron star with wind velocity $700 \mathrm{~km} \mathrm{~s}^{-1}$. The sample model parameters are shown in Table 1 . The effects of various parameters are shown by varying them one at a time, keeping others fixed. We show the effect of density parameter on $\mathrm{Fe}$ XXV and Fe XXVI lines by varying the density parameter alone keeping all other parameters fixed. Fig. 1 shows the absorption strength of Fe XXV and Fe XXVI lines as a function of energy for three different densities at the illuminated face of the accretion disc, $10^{10}, 10^{9}$ and $10^{8} \mathrm{~cm}^{-3}$, represented by blue, red and black solid lines, respectively. Absorption strengths of both Fe XXV and Fe XXVI lines change by a change in density. However in this case, a change in density does not produce any change in line width. For this sample model, above a particular density at the illuminated face of the accretion disc $\left(\sim 10^{10} \mathrm{~cm}^{-3}\right)$, the strength of Fe XXV line is much less than that of Fe XXVI. Below this density, the strength of Fe XXV is higher. This may be due to critical density effect. An increase in density at the illuminated face of the accretion disc from $10^{8} \mathrm{~cm}^{-3}$ to $10^{9} \mathrm{~cm}^{-3}$ increases the absorption for Fe XXVI line roughly by 100 $\%$ and decrease in absorption for line Fe XXV roughly by $33 \%$. Besides Fe XXV and Fe XXVI lines, very weak highly ionised lines from other species can also be found in this simple sample model.

\subsection{Effect of radiation field}

In all our models, we consider incident radiation to be consisted of both power-law continuum and blackbody continuum. In Fig. 2 we plot absorption strengths of Fe XXV and Fe XXVI lines as a function of energy with different ionisation parameters for the same sample model of LMXB (see
Table 1. Input parameters for our sample LMXB model.

\begin{tabular}{lr}
\hline Physical parameters & Values \\
\hline Power law: slope, $\log (\xi)$ & $-1.96,3.8$ \\
Blackbody: Temperature, $\log (\xi)$ & $10^{7} \mathrm{~K}, 3.2$ \\
Density $\mathrm{n}\left(\mathrm{r}_{0}\right)\left(\mathrm{cm}^{-3}\right)$ & $10^{9.5}$ \\
Metallicity & solar \\
Clump filling & 0.7 \\
Wind $\left(\mathrm{km} \mathrm{s}^{-1}\right)$ & 700 \\
Ionised column density $\left(\mathrm{cm}^{-2}\right)$ & 22.8 \\
\hline
\end{tabular}

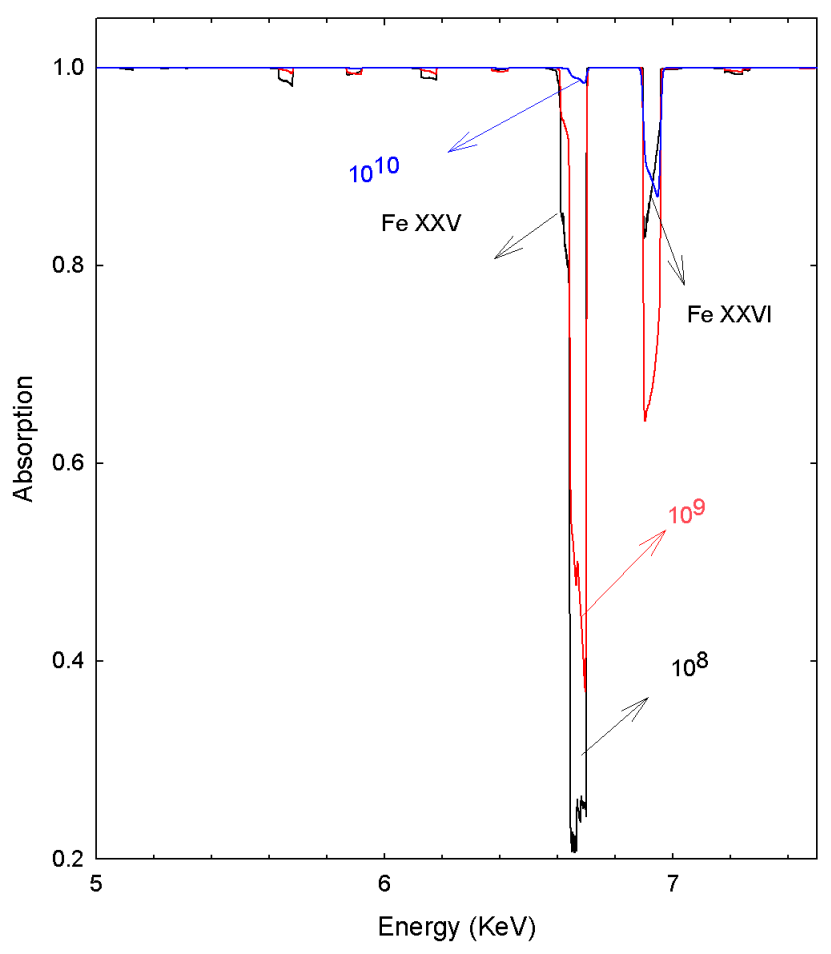

Figure 1. Change in relative absorption strength of Fe XXV and Fe XXVI lines with changing density are plotted as a function of energy in the energy range $5-7.5 \mathrm{keV}$. Here black, red and blue solid lines represent models with densities $10^{8} \mathrm{~cm}^{-3}, 10^{9}$ $\mathrm{cm}^{-3}, 10^{10} \mathrm{~cm}^{-3}$ at the illuminated face of the accretion disc, respectively (see section 2.2 ).

Table 1). Here we vary the ionisation parameter keeping all other parameters fixed. A decrease in ionisation parameter increases the line flux and line width of Fe XXV. A decrease in ionisation parameter from 3.8 to 3.3 increases the Fe XXV line absorption five times at the line centre. It has been observed by B05 and Díaz Trigo et al. (2006) that in the dipping phase of LMXB dippers, line flux and line width of Fe XXV increases. So Fig. 2 can also demonstrate that ionisation parameter is lower for the dipping phase compared to the persistent phase of LMXBs.

\subsection{Effect of wind}

Earlier modellers like B05, Díaz Trigo et al. (2006) and Juett \& Chakrabarty (2005) did not include disc wind in their 


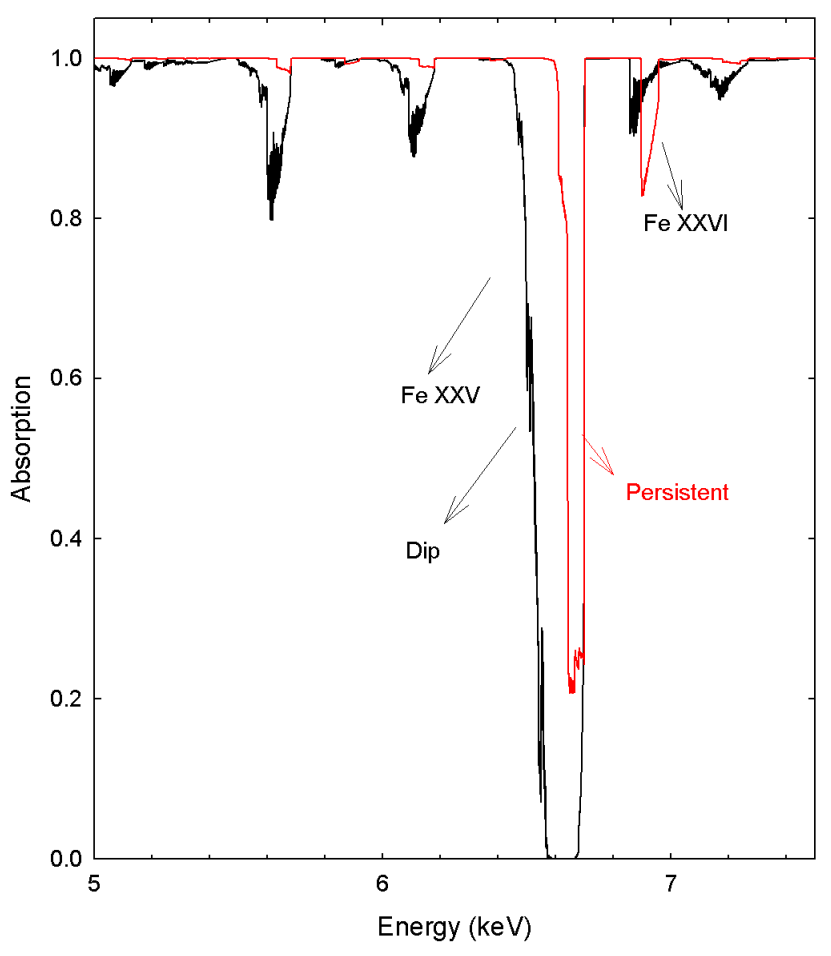

Figure 2. Change in relative absorption strength of Fe XXV and Fe XXVI lines are plotted as a function of energy in the energy range $5-7.5 \mathrm{keV}$. The red and black solid lines represent cases with higher and lower ionisation parameter, respectively (see section 2.3).

models. To make our model more realistic (D'Aì et al. 2014), we include disc wind and it is one of the important input parameters. Fig. 3 shows the effect of different wind velocities on the absorption strength of Fe XXV and Fe XXVI lines for the same sample model discussed earlier (see Table 1). Here we keep all the parameters of the sample model fixed and vary only the wind velocity. An increase in wind velocity from $1000 \mathrm{~km} \mathrm{~s}^{-1}$ to $1500 \mathrm{~km} \mathrm{~s}^{-1}$ increases both the line strengths by $\approx 20 \%$. In addition, metallicity and the clumpiness also effects the strength of the iron line absorption but to a lesser extent.

\subsection{Effect of magnetic field}

As we mentioned previously, in the presence of magnetic fields these absorption lines can get modified. Through the amount of change, one can estimate an upper limit on the strength of magnetic field in these accretion discs. Here we demonstrate that by comparing models with and without magnetic field.

Fig. 4 shows the effects of magnetic field strengths on Fe XXV and Fe XXVI lines. Here we represent models with no-magnetic field and $10^{3}$ Gauss magnetic field by the solid black and red lines, respectively. An increase in magnetic field to $10^{3} \mathrm{G}$ from null increases the line intensity of Fe XXV line approximately by $10 \%$ and decreases the line intensity of Fe XXVI line by $32 \%$. Higher magnetic field produces more significant effect. Thus, an upper limit of magnetic field can

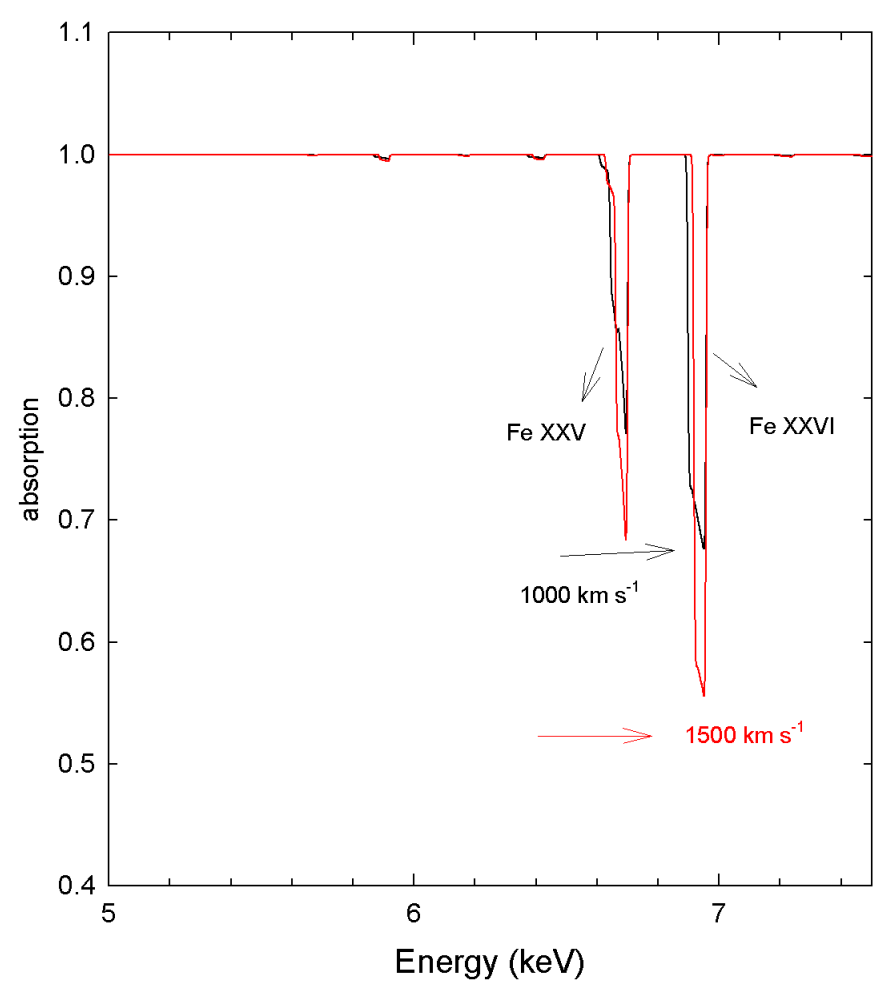

Figure 3. Change in relative absorption strength of Fe XXV and Fe XXVI lines are plotted as a function of energy in the energy range $5-7.5 \mathrm{keV}$ for disc wind velocities $1000 \mathrm{~km} \mathrm{~s}^{-1}$ (black solid line) and $1500 \mathrm{~km} \mathrm{~s}^{-1}$ (red solid line) respectively (see section 2.4).

be derived using the observed line fluxes of Fe XXV and Fe XXVI. The detectable amount of change in the absorption due to a few hundred Gauss magnetic field is the effect of change in temperature and pressure. A high magnetic field cools ionised gas through cyclotron emission and it is the dominant coolant. Our calculation shows that the gas temperature in the accretion disc decreases nearly by an order or factor of ten for the model with $10^{3}$ Gauss magnetic field compared to the zero magnetic field model.

\section{RESULTS}

In this section we present our results and elaborate on main findings. First we discuss results of GX $13+1$ in detail as it has the maximum number of observed lines. Then we briefly discuss the results for MXB 1659-298, $4 \mathrm{U} 1323-62$ and $4 \mathrm{U}$ 1916-053.

\section{$3.1 \quad$ GX $13+1$}

Here we model the persistent phase of GX $13+1$ reported by D'Aì et al. (2014). They observed absorption lines Fe XXV, Fe XXVI, Mg XII, Al XIII, Si XIV, S XVI, Ca XX and listed the line fluxes. We normalise line fluxes with respect to the weakest Al XIII line flux. D'Aì et al. (2014) have measured a 


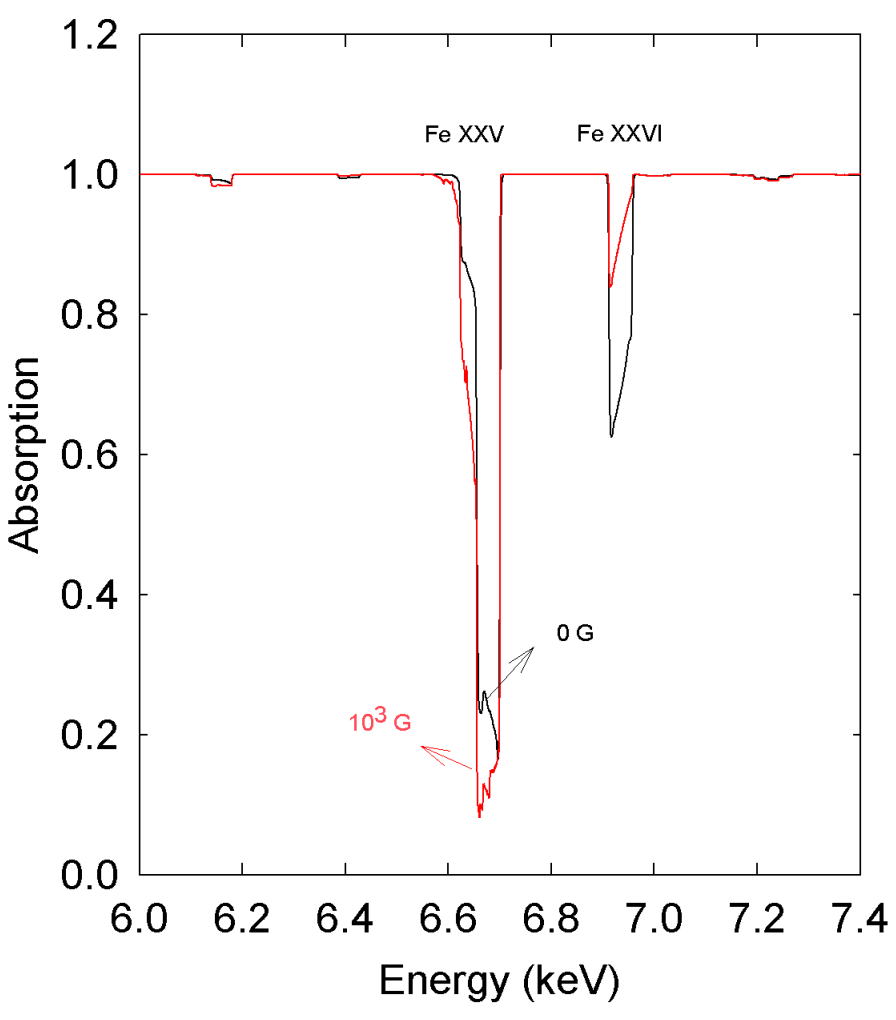

Figure 4. Effect of magnetic field on Fe XXV and Fe XXVI lines are plotted as a function of energy in the energy range 6- $7.4 \mathrm{keV}$. The solid black and red lines represent models with no-magnetic field and $10^{3}$ Gauss magnetic field, respectively (see section 2.5).

common blue-shift (weighted average $490 \mathrm{~km} \mathrm{~s}^{-1}$ ) for resonant transitions of $\mathrm{H}$-like ions coming from the accretion disc of GX $13+1$. We fix the wind velocity to $500 \mathrm{~km} \mathrm{~s}^{-1}$ (close to the observed value) for model calculations presented here for GX 13+1. The built-in CLOUDY optimisation program (see end of subsection 2.1) is widely used by astronomers (Ferland et al. 2013; Srianand \& Petitjean 2000; van Hoof et al. 2013; Rawlins et al. 2018) to match model predictions close to observed values. Applying the same approach, the optimisation models as mentioned earlier with different power law index values are performed, and we notice that the model with power index value -0.6 gives the best result. The gas phase abundances of the observed elements $\mathrm{Fe}, \mathrm{Mg}, \mathrm{Ca}, \mathrm{S}$ and $\mathrm{Si}$ are varied to match with observations and abundances of other elements are fixed to their solar values due to lack of observed lines. Dusts are not considered in this model as the temperature is much higher than the sublimation temperature of dusts. First, we calculate a best model without any magnetic field. In the next step, we add a tangled magnetic field in our model and vary its strength. The predicted line ratios start going beyond the observed range above $2000 \mathrm{G}$. The most affected lines are highly ionised $\mathrm{Fe}$ lines and the least affected lines are highly ionised $\mathrm{Mg}$ lines. As an example, we plot our model predictions as a function of magnetic field for lines Fe XXV and Fe XXVI in Fig.5. The predicted Fe XXV and Fe XXVI line ratios remain within the observed range for magnetic field less than $10^{3} \mathrm{G}$. We also show results for all the detected lines in
Table 2 for various magnetic field values. From our calculations, we can estimate a magnetic field upper limit of close to 2000 G. Note that our results do show (see Table 2 and Fig.5 ) that for a much higher magnetic field value, the predicted line ratios are far from the observed ranges. Normalisation with respect to a different line change the line ratios. But the overall physical conditions and the upper limit of magnetic field remain almost the same as the choice of normalisation does not change the system. Table 3 lists predicted physical parameters for our best model. Our predicted hydrogen density is $10^{12.1} \mathrm{~cm}^{-3}$ which is close to that predicted by D'Aì et al. (2014) who reported a density of $10^{12} \mathrm{~cm}^{-3}$. As mentioned earlier, we consider incident radiation field consisting of a power law continuum and a blackbody radiation. Earlier, Migliari et al. (2005) found the power index to be -0.6. Whereas, Paizis et al. (2006) have calculated a power index of $-2.8_{-1.33}^{+1.48}$. We try three power indexes $-0.6,-1$ and -2 . For our best model, the power law radiation has a spectral index -0.6 and the temperature of the blackbody radiation is $3 \times 10^{7} \mathrm{~K}$. Similar temperature for the blackbody radiation has been reported by others too. Our best ionisation parameters for these two radiations are 3.0 and 1.0 (as mentioned earlier), respectively. In general, radial thickness of the accretion disc from its illuminated face ranges from $10^{9} \mathrm{~cm}$ to $10^{11} \mathrm{~cm}$. Here, the best model predicts that the above mentioned lines originate near the edge of the disc, close to $1.6 \times 10^{11} \mathrm{~cm}$, and the radial thickness of the accretion disc from its illuminated face to be $10^{11} \mathrm{~cm}$. D'Aì et al. (2014) had mentioned a similar value of $1.4 \times 10^{11} \mathrm{~cm}$. Accretion disc is highly ionised and our predicted ionised column density is $10^{23.0} \mathrm{~cm}^{-2}$. Elemental abundances differ from their solar values with different amounts for this LMXB. Elemental abundances of $\mathrm{Ca}$ and $\mathrm{Si}$ are close to their respective solar values, whereas elemental abundance of $\mathrm{Fe}$ is more than its respective solar value. Table 4 compares the predicted and observed line flux ratios with respect to the weakest line Al XIII for our best model. All the predicted line flux ratios are within the observed range. Besides the lines mentioned here, our best model predicts Ar XVIII and Ni XXVIII lines at $3.735 \AA$ and $1.533 \AA$, respectively. These two lines are not reported by the observers. In our calculation we consider solar abundances for $\mathrm{Ar}$ and Ni. Lack of observation despite model prediction might mean that the abundances of $\mathrm{Ar}$ and $\mathrm{Ni}$ are less than their respective solar values. As mentioned earlier, we include a tangled magnetic field in our calculation. Comparing our model predictions and observed values, we predict an upper limit of magnetic field of strength $\approx 1000 \mathrm{G}$ in the accretion disc. Fig.6 shows the ranges of temperature and electron density across the accretion disc as a function of depth from its illuminated face. The solid and dashed lines represent temperature and electron density, respectively. The temperature in the disc ranges from $4.72 \times 10^{6} \mathrm{~K}$ to $4.24 \times 10^{6} \mathrm{~K}$, whereas the electron density ranges from $1.48 \times 10^{12} \mathrm{~cm}^{-3}$ to $5.6 \times 10^{11} \mathrm{~cm}^{-3}$. In CLOUDY, temperature is determined by solving heating and cooling balance. It includes all the important heating and cooling mechanisms self-consistently to determine the temperature accurately. In Fig.7 we plot the cooling fraction of important coolants across the accretion disc as a function of depth from the illuminated face of the accretion disc. Most of the cooling, roughly 60 percent is contributed by free-free cooling. Rest are coming from adiabatic cooling 
Table 2. Comparison of observed and predicted line flux ratios of LMXB GX 13+1 with different magnetic field strengths (in units of $\mathrm{G}$ ) using CLOUDY. Lines are normalised w.r.t Al XIII line.

\begin{tabular}{|c|c|c|c|c|c|}
\hline Lines & $\begin{array}{c}\text { Observed } \\
\text { range }\end{array}$ & $\begin{array}{l}\text { Model } \\
\mathrm{B}=0\end{array}$ & $\begin{array}{l}\text { Model } \\
\mathrm{B}=10^{2}\end{array}$ & $\begin{array}{l}\text { Model } \\
\mathrm{B}=10^{4}\end{array}$ & $\begin{array}{l}\text { Model } \\
\mathrm{B}=10^{5}\end{array}$ \\
\hline $\mathrm{Mg} \mathrm{XII}^{a}$ & $0.39-3.23$ & 2.96 & 2.95 & 3.47 & 5.16 \\
\hline $\mathrm{Mg} \mathrm{XII}^{b}$ & $0.54-3.22$ & 0.88 & 0.88 & 0.56 & 0.27 \\
\hline Si XIV ${ }^{c}$ & $2.02-7.47$ & 4.99 & 4.98 & 6.53 & 6.16 \\
\hline $\mathrm{S} X V I^{d}$ & $0.93-4.48$ & 1.86 & 1.86 & 1.41 & 0.79 \\
\hline Ca XX ${ }^{e}$ & $1.12-8.14$ & 2.49 & 2.49 & 1.27 & 0.02 \\
\hline Fe XXV $f$ & $3.91-17.92$ & 6.83 & 6.83 & 1.19 & $<0.005$ \\
\hline $\mathrm{Fe} \mathrm{XXVI}{ }^{g}$ & g $11.95-45.0$ & 15.92 & 15.89 & 2.71 & $<0.005$ \\
\hline $\mathrm{Fe} \mathrm{XXVI}^{h}$ & h $3.16-28$ & 3.20 & 3.20 & 0.59 & $<0.005$ \\
\hline
\end{tabular}

${ }^{a} 1.472 \mathrm{keV}, \quad{ }^{b} 1.744 \mathrm{keV}, \quad{ }^{c} 2.005 \mathrm{keV}, \quad{ }^{d} 2.621 \mathrm{keV}$,

${ }^{e} 4.105 \mathrm{keV}, \quad{ }^{f} 6.700 \mathrm{keV}, \quad{ }^{g} 6.966 \mathrm{keV}, \quad{ }^{h} 8.250 \mathrm{keV}$.

Table 3. Predicted model parameters of LMXB GX 13+1 using CLOUDY.

\begin{tabular}{lr}
\hline Physical parameters & Best values \\
\hline Power law: slope, $\log (\xi)$ & $-0.6,3.0$ \\
Blackbody: Temperature, $\log (\xi)$ & $3 \times 10^{7} \mathrm{~K}, 1$ \\
Density n $(\mathrm{H})\left(\mathrm{cm}^{-3}\right)$ & $10^{12.1}$ \\
Ionised column density $\left(\mathrm{cm}^{-2}\right)$ & 23.2 \\
Upper limit of magnetic field $(\mathrm{G})$ & 2000 \\
$\mathrm{Fe} / \mathrm{H}$ & -4.31 \\
$\mathrm{Mg} / \mathrm{H}$ & -4.64 \\
$\mathrm{Ca} / \mathrm{H}$ & -5.7 \\
$\mathrm{~S} / \mathrm{H}$ & -5.56 \\
$\mathrm{Si} / \mathrm{H}$ & -4.5 \\
\hline
\end{tabular}

Table 4. Comparison of observed and predicted best line flux ratios of LMXB GX 13+1 using CLOUDY. Lines are normalised w.r.t $\mathrm{Al}$ XIII line.

\begin{tabular}{lcr}
\hline Lines $E_{l a b}(\mathrm{kev})$ & Observed & Best model \\
\hline Mg XII (1.472) & $0.39-3.23$ & 2.96 \\
Mg XII (1.744) & $0.54-3.22$ & 0.88 \\
Si XIV (2.005) & $2.02-7.47$ & 4.99 \\
S XVI (2.621) & $0.93-4.48$ & 1.86 \\
Ca XX (4.105) & $1.117-8.139$ & 2.49 \\
Fe XXV (6.700) & $3.91-17.92$ & 6.83 \\
Fe XXVI (6.966) & $11.95-45.0$ & 15.92 \\
Fe XXVI (8.250) & $3.16-28$ & 3.2 \\
\hline
\end{tabular}

and Compton cooling, respectively. In Fig.8, we show the heating fraction as a function of depth from the illuminated face of the accretion disc. Most of the heating comes from Compton heating, which is again roughly 65 percent. The other important heating agents are heating by species Fe25 and Fe26, respectively.

\subsection{MXB 1659-298}

MXB 1659-298 is also a NS-LMXB which shows type-I Xray bursts. Recently Iaria et al. (2018b) have observed O VIII, Ne X, Fe XXV, Fe XXVI, Fe XXV and Fe XXVI lines for MXB 1659-298 using XMM-Newton, NuSTAR and Swift/XRT. We follow a similar model for MXB 1659-298

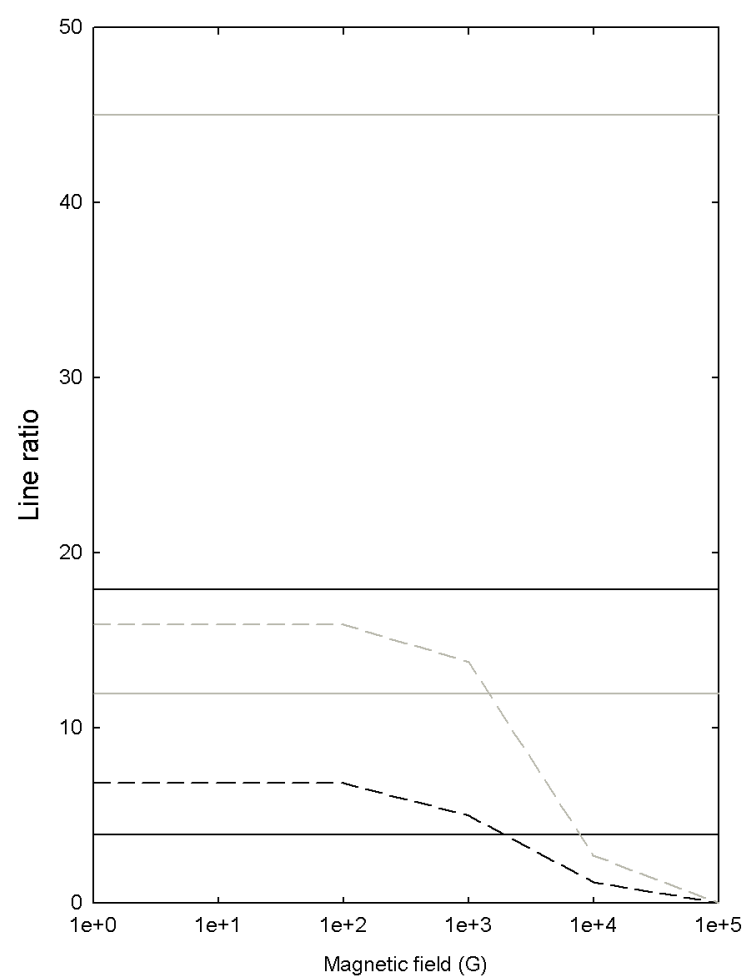

Figure 5. Predicted Fe XXV line ratio for GX $13+1$ is plotted as a function of magnetic field. The horizontal lines represent the observed range (see section 3.1). The black and gray lines represent data for Fe XXV and Fe XXVI, respectively.

like the one used for GX $13+1$. We consider the observed line fluxes reported by Iaria et al. (2018b) and use CLOUDY to model these lines to understand the underlying physical conditions. In this case, we normalise line fluxes with respect to the weakest O VIII line flux. Unlike GX $13+1$, the wind velocity is not detected unambiguously here. So, we keep wind velocity as a free parameter for the model of MXB 1659298. Tabel 5 and Table 6 list the best model parameters and predicted line flux ratios using CLOUDY. Our predicted hydrogen density is $10^{10.45} \mathrm{~cm}^{-3}$. For our best model, the power law radiation has a spectral index of -2.1 and the temperature of the blackbody radiation is $5 \times 10^{7} \mathrm{~K}$. The ionisation parameters for power-law and black body radiations are 2.94 and 1.66, respectively. Here, the best model predicts the radial expansion of the accretion disc from its illuminated face to be $1 \times 10^{11} \mathrm{~cm}$, whereas the predicted ionised column density is $10^{23} \mathrm{~cm}^{-2}$. Elemental abundances of $\mathrm{Fe}, \mathrm{O}$ and $\mathrm{Ne}$ are close to their solar values. All the predicted line flux ratios except the Fe XXVI $(8.250 \mathrm{keV})$ are within the observed range. Prediction for the Fe XXVI line does not improve even when we include all the iso-electronic levels of Fe. However, for completeness we show our predic- 


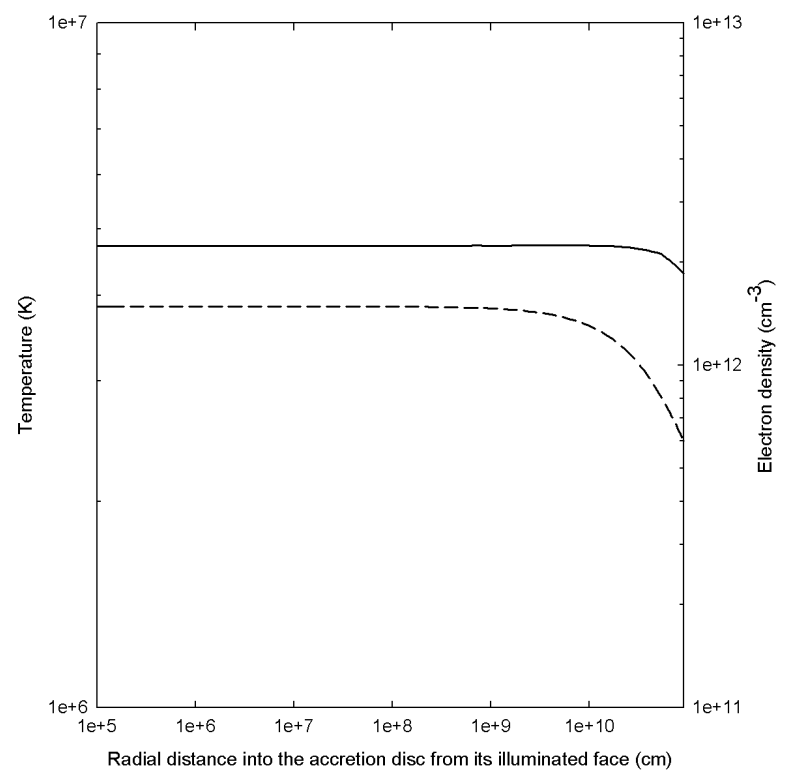

Figure 6. Temperature and electron density are plotted as a function of distance into the accretion disc. The solid and dashed lines represent temperature and electron density, respectively (see section 3.1.)

Table 5. Predicted model parameters of MXB 1659-298 using CLOUDY.

\begin{tabular}{lr}
\hline Physical parameters & Best values \\
\hline Power law: slope, $\log (\xi)$ & $-2.1,2.94$ \\
Blackbody: Temperature, $\log (\xi)$ & $5 \times 10^{7} \mathrm{~K}, 1.66$ \\
Density n $(\mathrm{H})\left(\mathrm{cm}^{-3}\right)$ & $10^{10.8}$ \\
Clump filling & 0.6 \\
Wind (km/sec) & 700 \\
Ionised column density $\left(\mathrm{cm}^{-2}\right)$ & 23.0 \\
Upper limit of Magnetic field $(\mathrm{G})$ & $\approx 1000$ \\
$\mathrm{Fe} / \mathrm{H}$ & -4.4 \\
$\mathrm{O} / \mathrm{H}$ & -4.32 \\
$\mathrm{Ne} / \mathrm{H}$ & -4.1 \\
\hline
\end{tabular}

tion for this line in Table 6. As mentioned earlier, in the final stage we include a tangled magnetic field in our calculation. We find that, with the upper limit magnetic field $\approx 1000 \mathrm{G}$, all the $\mathrm{Fe}$ line ratios exceed the observed ranges. No such drastic effect is observed for Ne X line.

\section{$3.34 \mathrm{U} \quad 1323-62$}

Here, we model the spectral lines of $4 \mathrm{U} 1323-62$. In this case, observed absorption fluxes of Fe XXV and Fe XXVI lines are taken from the panel A of Figure 2 of B05, and we try to match the flux ratios of these two lines (Fe XXV/Fe XXVI)

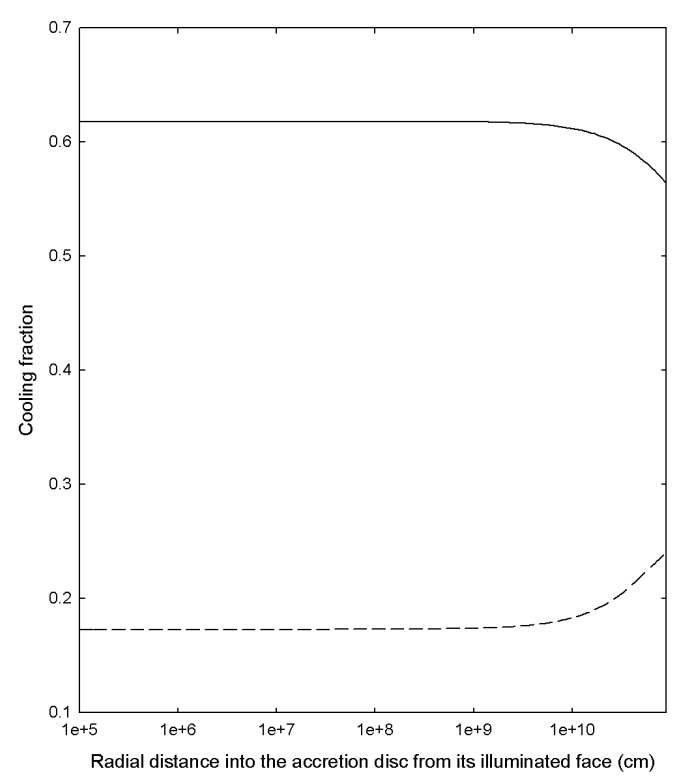

Figure 7. Cooling fractions for important coolants are plotted as a function of distance into the accretion disc. The solid and medium-dashed lines represent cooling by free-free transition and adiabatic cooling, respectively (see section 3.1 ).

Table 6. Comparison of observed and predicted line flux ratios of MXB 1659-298 using CLOUDY. Lines are normalised w.r.t O VIII line.

\begin{tabular}{lcr}
\hline Lines $E_{l a b}(\mathrm{kev})$ & Observed & Predicted \\
\hline Ne X (1.021) & $6.09-3.01$ & 4.98 \\
Fe XXV (6.700) & $28.7-16.10$ & 27.60 \\
Fe XXVI (6.966) & $36.23-21$ & 23.61 \\
Fe XXV (7.880) & $27.72-9.47$ & 10.64 \\
Fe XXVI (8.250) & $30.29-12.62$ & 4.05 \\
\hline
\end{tabular}

at the line centre. We vary all the parameters of our sample LMXB dipper model as discussed earlier and try to match the predicted absorption ratio of Fe XXV and Fe XXVI lines with the observed one. In Table 7 , list of predicted parameters for the best model of $4 \mathrm{U} 1323-62$ are shown. Our predicted ionisation parameters and ionised column densities in the persistent and dipping phases are very close to that predicted by $\mathrm{B} 05$. The best model predicts that the metallicity is sub-solar and clumps are present in the gaseous disc. This sub-solar metallicity may indicate the metallicity of the donor star. Earlier, Zolotukhin et al. (2010); Gambino et al. (2016) had estimated the mass of the donor star to be $0.28 \pm 0.03$ times solar mass but did not discuss about its metallicity. We predict that wind velocity, density and metallicity remain to be almost the same for both the per- 


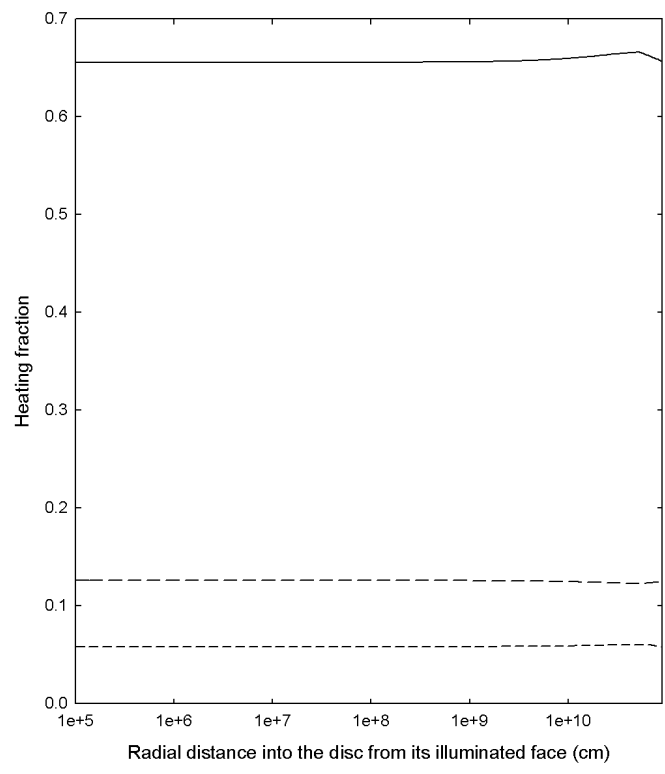

Figure 8. Heating fractions for important heating sources are plotted as a function of distance into the gaseous disc. The solid, medium-dashed and short-dashed lines represent Compton heating and heating by species $\mathrm{Fe} 25$ and $\mathrm{Fe} 24$ respectively (see section 3.1.)

sistent and dip phases, whereas the ionisation parameter and the ionised column density are the only parameters that differ in persistent and dip phases. Earlier, B05 used more than one incident radiation fields in their modelling. We also predict an incident radiation field consisting of a blackbody radiation of temperature $10^{7} \mathrm{~K}$ and Comptonised radiation with power law index -1.99 . Here wind velocity is $700 \mathrm{~km}$ $\mathrm{s}^{-1}$. Note that Ueda et al. (2005) also observed similar wind velocities for Galactic LMXBs earlier.

Fig. 9 shows effects of various magnetic field strengths on these two lines for the persistent phase of $4 \mathrm{U} 1323-62$. We predict that increasing the magnetic field by a small amount changes both the line strength and line width. Comparing our simulated spectra and the observed data, we estimate the upper limit on the strength of prevailing magnetic field in the accretion disc of LMXB $4 \mathrm{U} 1323-62$ to be about 1000 G. Here, we determine various physical parameters by only matching the observed flux ratio of Fe XXV and Fe XXVI lines at line centre.

\subsection{XB 1916-053}

Here we discuss on the last source, XB 1916-053. The observed data for this LMXB are taken from Iaria et al. (2006). Iaria et al. (2006) have observed Ne X, Fe XXV, Fe XXVI, Mg XII, Si XIV and S XVI for XB 1916-053 using Chandra
Table 7. Predicted physical parameters by CLOUDY modelling of $4 \mathrm{U} 1323-62$.

\begin{tabular}{|c|c|c|}
\hline Physical parameters & Persistent & Dip \\
\hline Power law: slope, $\log (\xi)$ & $-1.99,3.8$ & $-1.99,3.3$ \\
\hline Blackbody: Temperature, $\log (\xi)$ & $10^{7} \mathrm{~K}, 3.1$ & $10^{7} \mathrm{~K}, 2.5$ \\
\hline Density $\mathrm{n}\left(\mathrm{r}_{0}\right)\left(\mathrm{cm}^{-3}\right)$ & $10^{9.3}$ & $10^{9.3}$ \\
\hline Metallicity & 0.5 solar & 0.5 solar \\
\hline Clump filling & 0.5 & 0.5 \\
\hline Wind $\left(\mathrm{km} \mathrm{s}^{-1}\right)$ & 700 & 700 \\
\hline Ionised column density $\left(\mathrm{cm}^{-2}\right)$ & 22.8 & 23.3 \\
\hline Upper limit of disc magnetic field & $\leq$ few $100 \mathrm{G}$ & - \\
\hline
\end{tabular}

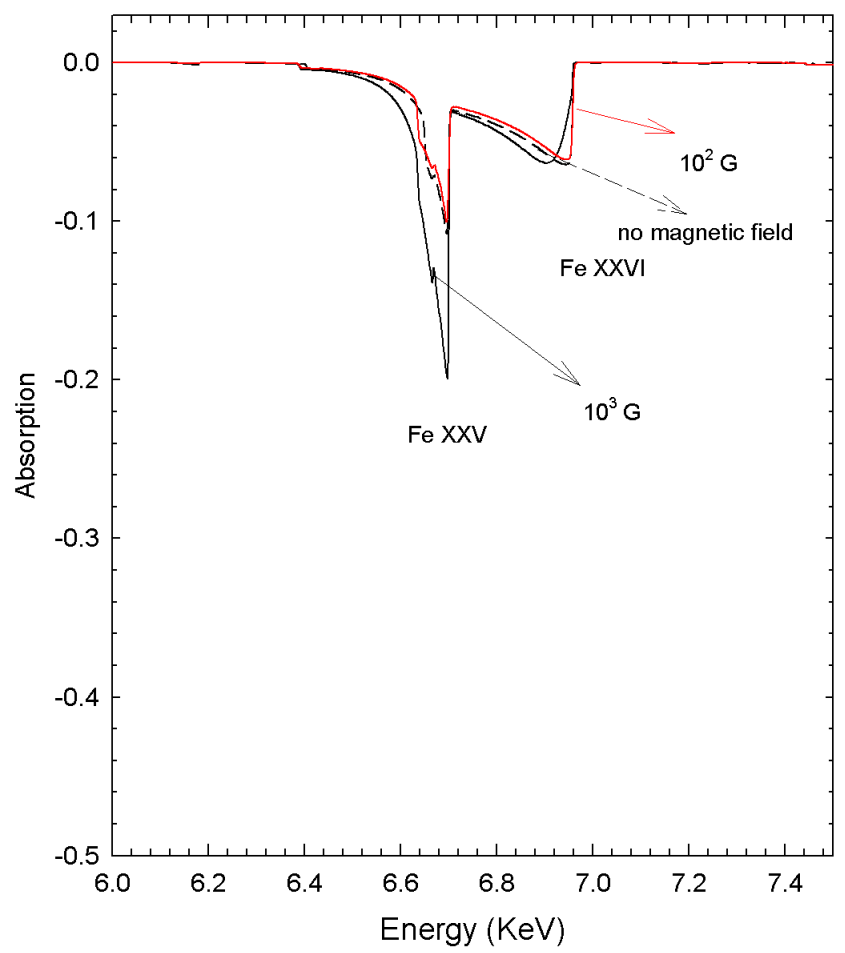

Figure 9. Effect of magnetic field on the modelled spectra for $4 \mathrm{U}$ 1323-62 in persistent phase is plotted as a function of energy in the energy range $6-7.5 \mathrm{keV}$. The medium-dashed black line, solid red line and solid black line represents models with no-magnetic field, $10^{2}$ and $10^{3}$ Gauss magnetic field, respectively (see section 3.3). The other parameters are listed in Table 7

satellite. We follow the similar model used for GX $13+1$. We consider the observed line fluxes reported by Iaria et al. (2006) and use CLOUDY to model these lines to understand the underlying physical conditions. In this case, we normalise line fluxes with respect to the weakest Mg XII line flux. The wind velocity is not detected unambiguously here. So, we keep wind velocity as a free parameter for the model of XB 1916-053. Tabel 8 and Table 9 list the best model parameters and predicted line flux ratios using CLOUDY. We ran models with three power law radiation spectral indices, -.5, -1.5 and -2 . For our best model, the power law radiation has a spectral index of -1.5 and the temperature of the blackbody radiation is $2.8 \times 10^{7} \mathrm{~K}$. The ionisation parame- 
Table 8. Predicted physical parameters by CLOUDY modelling of XB 1916-053.

\begin{tabular}{lr}
\hline Physical parameters & Best values \\
\hline Power law: slope, $\log (\xi)$ & $-1.5,3.0$ \\
Blackbody: Temperature, $\log (\xi)$ & $10^{7} \mathrm{~K}, 1.6$ \\
Density n( $\left.\mathrm{r}_{0}\right)\left(\mathrm{cm}^{-3}\right)$ & $10^{11.77}$ \\
Clump filling & 0.7 \\
Wind $\left(\mathrm{km} \mathrm{s}^{-1}\right)$ & 500 \\
$\mathrm{Fe} / \mathrm{H}$ & -4.170 \\
$\mathrm{Mg} / \mathrm{H}$ & -5.037 \\
$\mathrm{Ne} / \mathrm{H}$ & -4.038 \\
$\mathrm{~S} / \mathrm{H}$ & -5.250 \\
$\mathrm{Si} / \mathrm{H}$ & -4.856 \\
$\mathrm{Ionised}$ column density $\left(\mathrm{cm}^{-2}\right)$ & 22.53 \\
$\mathrm{Upper}$ limit of the disc magnetic field & $\approx 1000 \mathrm{G}$ \\
\hline
\end{tabular}

Table 9. Comparison of observed and predicted best line flux ratios of XB 1916-053 using CLOUDY. Lines are normalised w.r.t Mg XII line.

\begin{tabular}{lcr}
\hline Lines $E_{l a b}(\mathrm{kev})$ & Observed & Predicted \\
\hline Si XIV (2.005) & $0.98-2.39$ & 1.59 \\
S XVI (2.621) & $0.64-2.05$ & 1.03 \\
Fe XXV (6.700) & $0.49-2.11$ & 1.18 \\
Fe XXVI (8.250) & $1.45-4.30$ & 1.83 \\
Ne X (1.021) & $1.79-5.1$ & 2.76 \\
\hline
\end{tabular}

ters for power-law and black body radiations are 3.0 and 1.6, respectively. One can observe that our predicted ionisation parameters and ionised column densities in the persistent phase are very close to that predicted by Díaz Trigo et al. (2006). However, (Iaria et al. 2006) have found a higher value for the ionization parameter, 4.15. Our predicted hydrogen density is $10^{11.77} \mathrm{~cm}^{-3}$. Here, the best model predicts that the above mentioned lines originate near the edge of the disc, close to $7.4 \times 10^{10} \mathrm{~cm}$, and the radial expansion of the emitting region of these lines to be $10^{11} \mathrm{~cm}$. Iaria et al. (2006) have also argued that these lines are produced nearer to $4 \times 10^{10} \mathrm{~cm}$. The predicted ionised column density is $10^{22.53}$ $\mathrm{cm}^{-2}$. Elemental abundances of $\mathrm{Fe}, \mathrm{Mg}, \mathrm{S}, \mathrm{Si}$ are sub-solar except Ne. All the predicted line flux ratios are within the observed range. However, like the earlier cases, in the final stage we include a tangled magnetic field in our calculation. We find that with the strength of the magnetic field greater than $2 \times 10^{3} \mathrm{G}$, all the Fe line ratios exceed the observed ranges. No such drastic effect is observed for Ne X, Si XIV, $\mathrm{S} X V I$ lines. We plot our model predictions as a function of magnetic field for lines Fe XXV and Fe XXVI in Fig. [10]. Comparing our simulated spectra and the observed data, we estimate the upper limit on the strength of prevailing magnetic field in the accretion disc of XB 1916-053 to be close to $1000 \mathrm{G}$.

\section{CONCLUSIONS}

We use the spectral synthesis code CLOUDY (Ferland et al. 2013, 2017) to do a detailed modelling of the observed X-ray lines of the low-mass X-ray binaries, GX 13+1, MXB 1659-298, 4U 1323-62, XB 1916-053 and determine various

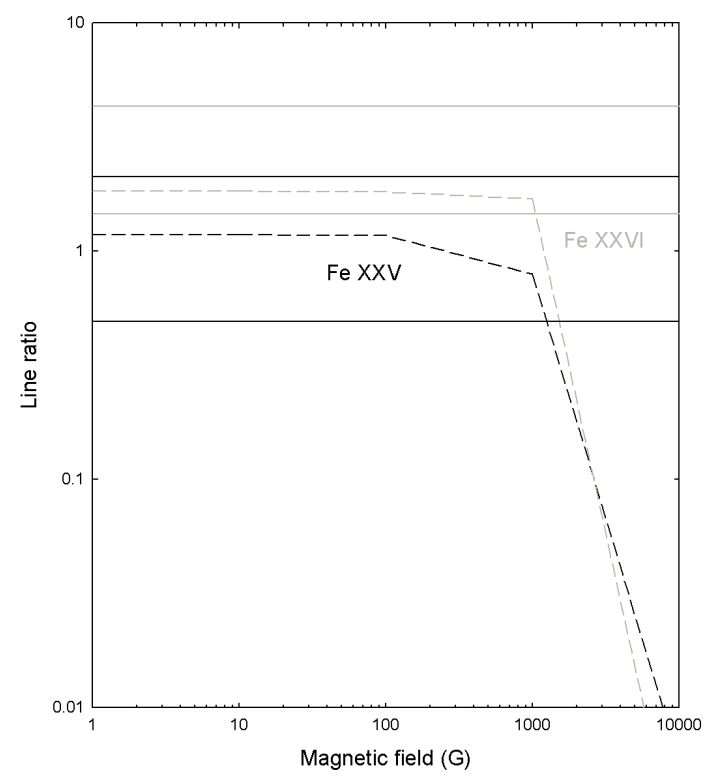

Figure 10. Predicted Fe line ratios for XB 1916-053 is plotted as a function of magnetic field. The horizontal lines represent the observed range (see section 3.4). The black and gray lines represent data for Fe XXV and Fe XXVI, respectively.

underlying physical parameters of these LMXBs. In addition to that, we developed a method to estimate an upper limit on the magnetic field of the associated accretion discs by studying these lines. We assume that the accretion disc has a geometry of a constant-pressure, clumpy, highly-ionised gaseous disc around a 1.4 solar mass neutron star with some velocity wind. A rotating disc around a 1.4 solar mass neutron star model prediction matches better with the observed values. In all the models, incident radiation is composed of both thermal blackbody and a single power law radiation. Our best model calculations predict almost all the line flux ratios within the observed range. Our main conclusions from this work are listed below:

- All the LMXBs considered here consist of highly ionised accretion discs. Our best models predict the radial extend of the accretion discs from their illuminated faces to be close to $10^{11} \mathrm{~cm}$.

- Total hydrogen density, consisting of ionised and atomic hydrogen together with all the hydrogen bearing molecules, of the gaseous accretion disc for these four LMXBs spans in the range of $10^{9.3} \mathrm{~cm}^{-3}$ to $10^{12.1} \mathrm{~cm}^{-3}$.

- We assume that the incident radiation consists of a blackbody radiation and a Comptonised continuum. The temperature of blackbody radiation ranges between $1 \times 10^{7} \mathrm{~K}$ to $5 \times 10^{7} \mathrm{~K}$. Spectral indices are found to be close to -2 for the three LMXBs, MXB 1659-298, 4U 1323-62. XB 1916053 has a spectral index of -1.5 . Whereas, spectral index is close to -0.6 for GX $13+1$. Similarly, the ionisation parameter for the persistent phase of these LMXBs ranges between 
2.94 to 3.8 for power law radiation and 1 to 3.1 for the blackbody radiation.

- Ionisation parameters are higher for persistent phases than that of dipping phases.

- In our models we use the observed wind velocity, whenever available. Otherwise we treat it as a free parameter. The wind velocity for the four LMXBs come out to be between $400 \mathrm{~km} \mathrm{~s}^{-1}$ to $700 \mathrm{~km} \mathrm{~s}^{-1}$.

- For these four LMXBs, most of the heating is contributed by Compton heating.

- We find that, most of the cooling is contributed by freefree transitions in these four LMXBs.

- Elemental abundances of $\mathrm{Ca}$ and $\mathrm{Si}$ are close to their respective solar values but the elemental abundance of $\mathrm{Fe}$ is more than its solar value for GX 13+1. 4U 1323-62 and XB 1916-053 have sub-solar metallicity (except Ne).

- We predict presence of clumps in the accretion discs.

- The predicted ionised column density in the persistent phase for the four LMXBs are between $10^{22.53} \mathrm{~cm}^{-2}$ to $10^{23.2}$ $\mathrm{cm}^{-2}$.

- Ionised column density is lower in the persistent phases than that of in the dipping phases.

- We predict an upper limit for the strength of prevailing magnetic field in the accretion discs to be $\sim 1000 \mathrm{G}$ which could be verified by future X-ray polarimetric observations.

\section{ACKNOWLEDGEMENTS}

Gargi Shaw acknowledges WOS-A grant from Department of Science and Technology (SR/WOS-A/PM-9/2017). We thank to the referee for valuable comments and suggestions.

\section{REFERENCES}

Bandyopadhyay R. M., Shahbaz T., Charles P. A., \& Naylor T., 1999, MNRAS, 306, 417

Balbus S. A. \& Hawley J. F., 1994, MNRAS, 266, 769

Bianchi S., Giorgio M., Fabrizio N., et al., 2005, MNRAS, 357, 599

Boirin L., Mendez M., Diaz Trigo M., et al., 2005, A\&A, 436, 195 [B05]

D’Aì A., Iaria R., Salvo T. Di., Riggio A., Burderi L., \& Robba N. R., 2014, A\&A, 564, 62

Díaz Trigo M., \& Boirin Laurence, 2013, AcPol, 53, 659

Díaz Trigo M., Sidoli L., Boirin L., \& Parmar A. N., 2012, A\&A, 543, A50

Díaz Trigo M., Parmar A. N., Boirin L., et al., 2006, A\&A, 445, 179

Duncan K. Galloway, Alishan N. Ajamyan, James Upjohn et al., 2016, MNRAS, 461, 384

Ferland G. J., Porter R. L., van Hoof P. A. M., et al., 2013, RMxAA, 49, 137

Ferland G. J., Chatzikos M., Guzman F., et al., 2017, RMxAA, 53,385

Fleischman J. R., 1985, AA, 153, 106

Foulkaes S. B., Haswell Carole A., Murray, James R., 2010, MNRAS, 401, 1275

Gambino A. F. et al., 2016, A\&A, 589, 34

Homer L., Charles P. A., Hakala P., Muhli P., et al., 2001, MNRAS, 322, 827

Iaria R., DiSalvo T., Lavagetto G., et al., 2006, ApJ, 647, 1341

Iaria R., DiSalvo T., Burderi L., Riggio A., D'Ai, A. \& Robba N. R., 2013, AA, 561, 99
Iaria R. et al., 2018a, MNRAS, 473, 3490

Iaria R. et al., 2018b, ArXiv Astrophysics e-prints, arXiv: 1807.11431

Juett Adrienne M., \& Chakrabarty Deepto, 2005, ApJ,627, 926J

Juhan Frank, Andrew King \& Derek Raine, 2002, Accretion Power in Astrophysics, New York, NY, Cambridge University Press.

Kallman T., \& Bautista M., 2001, ApJS, 133, 221

Lamb D. Q., Masters A. R., 1979,ApJ, 234, 117L

Landi E., Del Zanna G., Young P. R., Dere K. P., Mason H. E., $2012,, 744,99$

Lewin W. H. G., Hoffman J. A., Doty J., Liller W., 1976, IAU Circ. 2994

Mc Kinney J. C. \& Narayan R., 2007, MNRAS, 375, 513

Matt G., Gabian A. C., Ross R. R., 1993, MNRAS, 262, 179

Migliari S., Fender R. P., van der Klis M., 2005, MNRAS, 363, 112

Naso L., Kluzniak W., Miller J. C., 2013, MNRAS, 435, 2633

Niellsen M.T.B., Gilfanov M., 2015, MNRAS, 453, 292

Paizis A. et al., 2006, A\&A, 459, 187

Parfrey K., Spitkovsky A., Beloborodov A. M., 2016, ApJ, 822,33

Porter R. L., Ferland G. J., Storey P. J., \& Detisch M. J., 2012, MNRAS, 425, L28

Rawlins K., Srianand R., Shaw G., et al., 2018, MNRAS, 481, 2083

Russel D. M. \& Fender R. P., 2018, MNRAS, 387, 713

Schoier F. L., van der Tak F. F. S., van Dishoeck E. F., \& Black J. H., 2005, AA, 432, 369

Silantev N. A., Gnedin Yu. N., Buliga S.D. et al., 2013, Astrophysical bulletin, 68,14

Shapiro S.L. \& Teukolski S.A., 1984 Black Holes, White Dwarfs and Neutron Stars, Wiley-Interscience, New York

Shaw G., Ferland G. J., Abel N. P., et al., 2005, ApJ, 624, 794

Sobolev V. V., 1957, sva, 1, 678

R. Srianand \& P. Petitjean., 2000, A\&A, 357, 414

Tanaka Y. 1997, Ginga Memorial Symposium, ISAS, F. Makino \& F. Nagase.

Tauris T. M., \& van den Heuvel E. P. J., 2006, Compact Stellar X-Ray Sources, ed. W. Lewin \& M. van der Klis (Cambridge:Cambridge Univ. Press), 623

Ueda Y., Mitsuda K., Marakami H. et al., 2005, ApJ, 620, 274

van der Klis M., Jansen F., van Paradijs J. et al., 1985, Nature, 316, 225

van Hoof P. A. M., 1997, PhD thesis, Rijksuniversiteit Groningen van Hoof P. A. M. et al., 2013, A $\$$ A, 560, 7

White N. E., \& Swank J. H., 1982, ApJ, 253, L61

Younes G., Boirin L., \& Sabra B., 2009, A\&A, 502, 905

Zolotukhin I. Y., Revnivtsev M. G., Shakura N. I., 2010, MNRAS, 401, L1

This paper has been typeset from a $\mathrm{T}_{\mathrm{E}} \mathrm{X} / \mathrm{LAT}_{\mathrm{E}} \mathrm{X}$ file prepared by the author. 